\title{
SOME INTERESTING DESIGN ASPECTS FOR TALL RC CHIMNEYS AND BUILDINGS
}

\author{
S.N.Manohar
}

Consulting Engineer, Pune

\begin{abstract}
In recent decades, the heights of industrial chimneys and urban residential buildings have increased rapidly. Primarily, the former can be attributed to the need to effectively disperse ever increasing volume of pollutants under stricter pollution norms and the latter because of the steep increase in footprint land costs. As a parallel development, the increase in quality and strength of concrete has led to reduction in member sizes and thereby an increase in slenderness of these structures. While there are important differences between the behaviour of these two types of slender structures, there are also some similarities. This paper emphasizes the behaviour of tall slender chimney structures and presents the impact of dynamic wind effects and enhancement caused by correlation of wind forces due to small protrusions over the chimney height. This paper describes the manner of reducing the dynamic effect of wind on chimneys and how similar effect can be achieved for buildings with the help of architectural features.
\end{abstract}

Keywords: Chimney, Dynamic Effects, Pollution Dispersion, Tall Chimneys, Turbulence $* * *$

Wind is essentially the large scale movement of free air due to thermal currents. It plays an important role in the design of slender chimneys and buildings, particularly because it exerts static and dynamic forces whose effects are significant. The response of the structure to wind action is conveniently divided into those motions which are along wind and those which are cross wind but it may be noted that this distribution of convenience really relates to the forcing mechanism rather than the response. This paper deals primarily with dynamic effects.

Tall chimneys are line - like structures being exposed to dynamic wind loads. One major difficulty is to obtain dependable data concerning wind speeds and their direction. Dynamic effects of wind on the structure depend on wind speed, its turbulence (Fig 1), the chosen return period, the surroundings (Fig 2) etc. Similarly there are too many variables involved in assessing seismic loads. While codes do provide a guide to evaluate these forces; any deviation from the predicted pattern can have a major impact on the stresses in a chimney shell. In addition, there is the uncertainty regarding behaviour of a concrete shell under reverse cycle loading during a seismic event. Hence designers often opt for a conservative elastic design. Researchers have pointed out that this multi - degree of freedom system in the inelastic range can tolerate substantially higher seismic hazard than that required to cause the elastic moment demand. Due to this factor, coupled with flexural overstrength, the design shear forces increase under capacity design criteria.

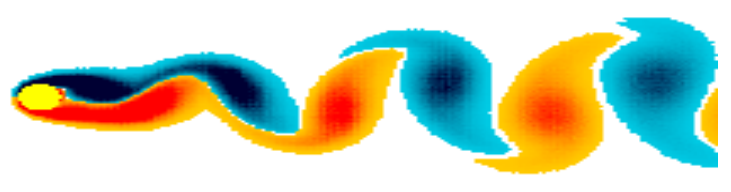

Fig. 1 Turbulence

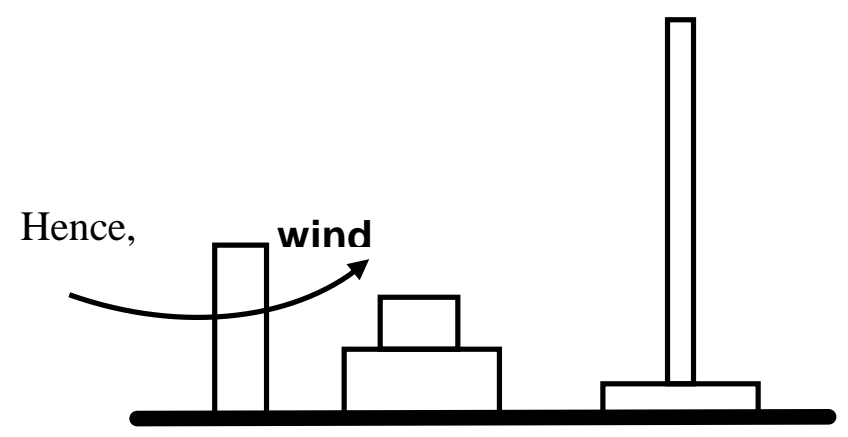

Fig.2 Effect ion Surroundings

Wind loads can be considered as a combination of steady and fluctuating along-wind loads. In addition, as wind flows past this bluff body, vortices are released alternately from opposite sides of a chimney. This generates an oscillatory aerodynamic loading (lift) on the chimney. The discrete velocity at which this occurs, can be predicted by the Strouhal number. However, during shedding of vortices, non - linear interaction occurs between the structure's motion and the wake. As a result, the wake may lock-in with the structures oscillation frequency over a large velocity range that increases as the structural damping decreases. This phenomenon can cause significant vibrations. 
To prevent lock-in due to vortex excitation, it is common to provide discrete strakes on RC chimneys. The optimum value for extent of protrusions, their pitch and interval between discrete strakes is a matter of debate. Similarly, at the right wind velocity in the right direction impinging on a tall building with the right slenderness; such a structure can also experience lateral forces. To prevent their lock-in; the architectural facade of a building can be sculptured so as to break such a formation (Fig 3)

It is well neigh impossible to accurately predict these wind effects precisely by analytical procedures because of wind's uncertain variability. In addition, generalized empirical methods of predicting cross wind response of slender structures have been difficult to formulate even assuming total dependence on wake excitation because of the effects of turbulence, operating reduced frequency range, structural damping, upstream interference etc. Despite significant advances in computational capabilities in recent years, the mathematical formulation to determine fluidstructure interaction has eluded numerical solutions for flow around structures. Thus, presently wind tunnels remain the most effective means of estimating wind effects on slender structures such as tall chimneys and buildings. This is so, in spite of the problems in simulation of $R_{e}$, scaling of wind, blockage effect in a wind tunnel etc.

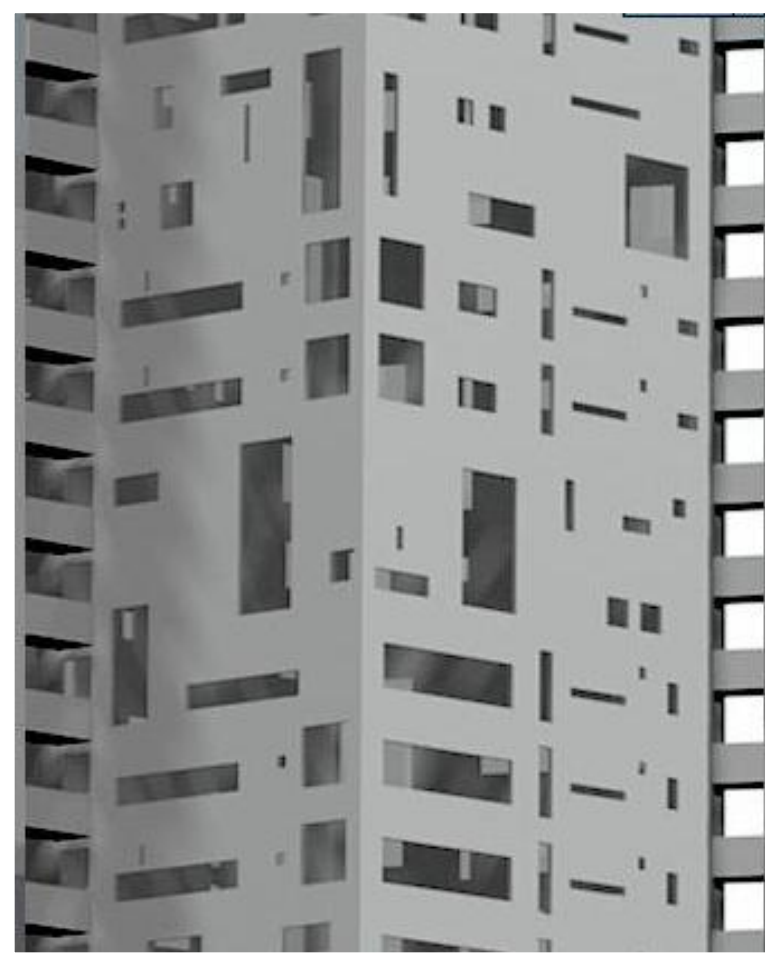

Fig. 3 Aerodynamics in Design

In this regard, an interesting effect was observed when we tested an aero-elastic model of a $275 \mathrm{~m}$ chimney model in a wind tunnel facility in Australia. The ladder rungs (along with their safety cage) running up the external face of the chimney was also modeled. We rotated the chimney in the wind tunnel and observed that when the ladder on the model was at an angle of about $120^{\circ}$ from the wind direction (Fig 4) there was a sharp increase in the mechanically admitted wind load on the chimney. Even after shifting the ladder location, the peak effect occurred at the above angle between the wind direction and the altered location of the ladder. This effect was noticed repeatedly. We did not have a convincing reason for this behaviour and surmised that it could be so because of a lock-in effect. At site, the ladder location was moved away from forming a $120^{\circ}$ angle with the predominant wind direction.

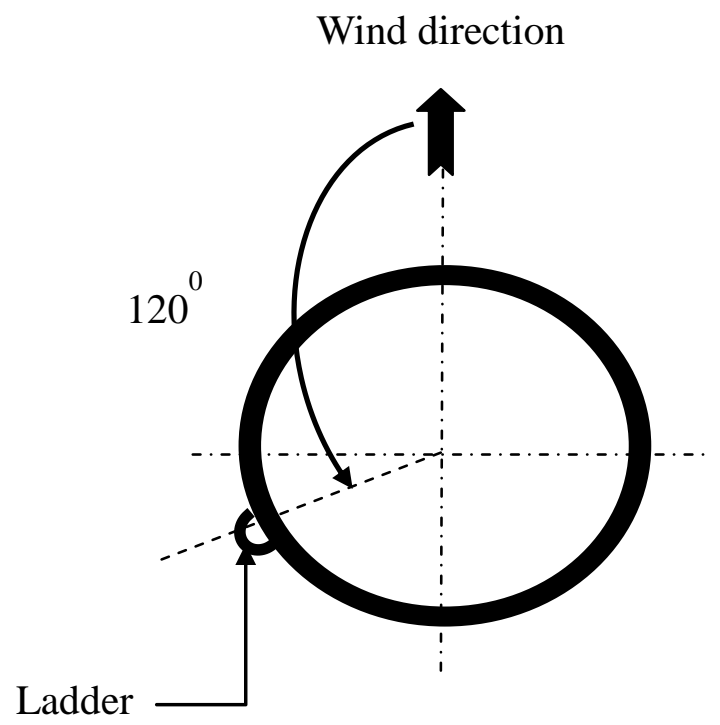

Fig. 4 Chimney Model

A comprehensive soil investigation should be undertaking. Under predominant wind loads, the foundation tilt needs to be evaluated for chimneys exceeding $400 \mathrm{~m}$ in height and the resulting $\mathrm{p}-\Delta$ effect and its impact on stresses. Many outstanding papers have been published regarding the need to consider soil structure interaction (SSI) while designing tall buildings. The same applies to chimneys. However, there is always the question of reliability of subsoil design parameters. Secondly, the results of such an analysis generally show that SSI has a significant effect for chimneys that rest on loose and medium sand. In such cases the practicing engineer normally opts for a piled foundation.

When analyzing a system with a large number of degrees of freedom using modal analysis technique, one needs to decide on the number of modes to consider in order to conserve computational effort. At the same time, adequate number of modes need to be included such that the evaluated response adequately reflects the true behaviour of the system. For this purpose, IS 1893 requires that during analysis, $90 \%$ of the modal mass should be captured in each orthogonal direction. Normally the desired result is achieved by considering the first few modes of vibration of a tall 
building. In some cases, however, it may be difficult to achieve this even when a reasonably large number of modes are considered or when the response of a dominant mass is controlled by a modal frequency that is at or above the rigid behaviour frequency. A typical example is when very stiff retaining walls of a basement form a part of the frame to be analysed (Fig5).

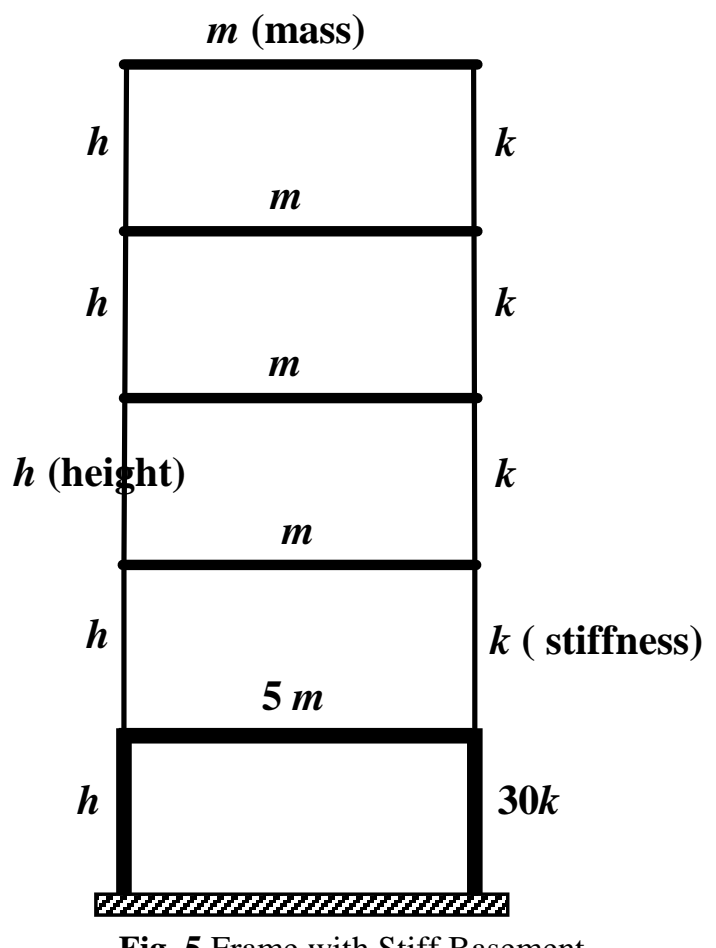

Fig. 5 Frame with Stiff Basement

In such cases, a technique known broadly as the missing mass correction method can be employed. It is a computational approach that is efficient and accurate.

The concept of this method is to undertake a modal analysis for all modes with frequencies below the zero period which is commonly taken as $33 \mathrm{~Hz}$. For these modes, the sum of participating modal masses at each storey is evaluated. These values for each floor are subtracted from the lumped masses at corresponding floor levels to arrive at missing masses at individual floors. The response of these missing masses is then obtained as if they will respond with a rigid body frequency i.e. the forces at a floor level will be the missing mass multiplied by peak ground acceleration in the acceleration spectrum. This response is then added to the earlier modal responses to arrive at the total response.

A staircase is a crucial means of escape from a tall building during a seismic event. It has to be ensured that supports of these stairs are so designed that the staircase does not form a part of the rigid building framework as otherwise the stair members will attract heavy compressive and tensile axial forces (Fig 6). To fulfill this requirement, the supports along one end need to be moveable.

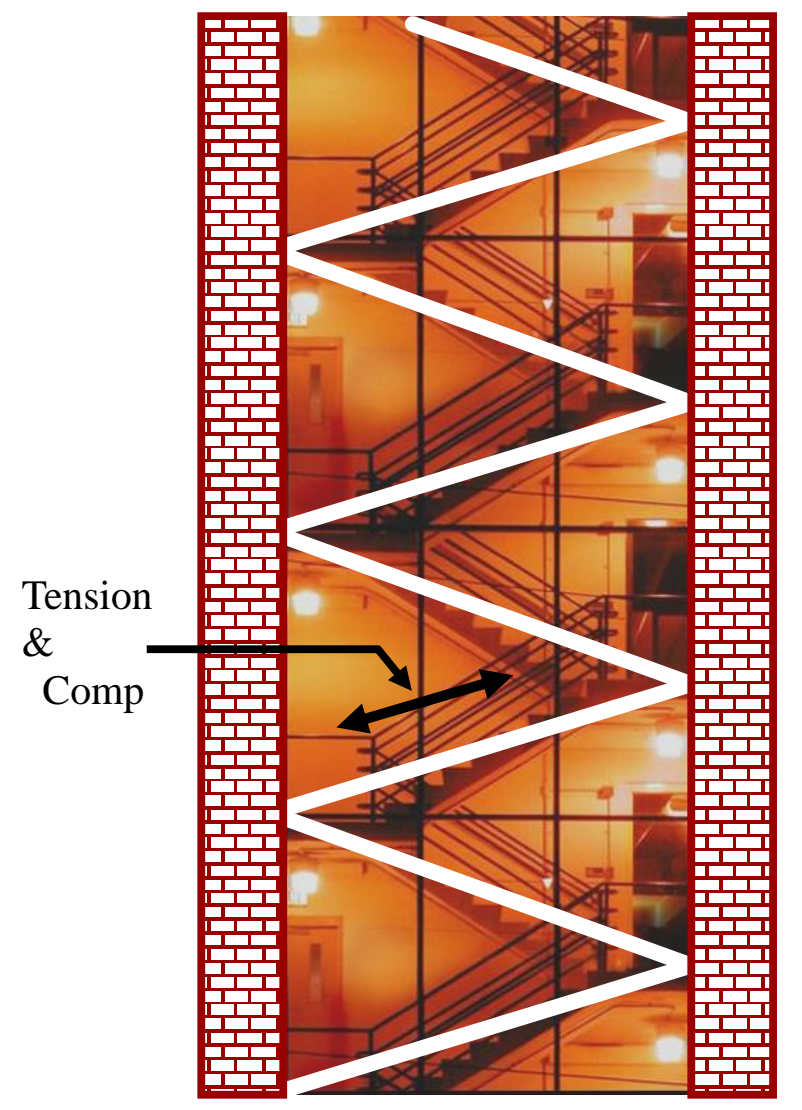

Fig 6. Staircase Framing

There is often a debate as to whether wind or earthquake controls the stresses in a particular building. Some decades ago, the answer to this question used to be simple; based generally on the building height and knowledge of wind and earthquake zones in which the structure was located. However with the advent of tall buildings, and sophistications in wind and seismic design by considering dynamic effects and introduction of capacity based design principles; the answer to this question is not simple anymore. The parameters for comparing the effects of these two types of loads are no longer identical.

\section{CONCLUSIONS}

Slender srtructures need to be analyzed for dynamic effects and wind tunnel studies should be entrusted only to experts in the field.

\section{BIOGRAPHY}

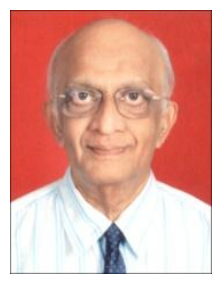

S N Manohar is a very senior civil engineer with over five decades of experience who lives in Pune. He is a noted expert on the design and construction of Tall Chimneys and has authored a very popular book on the subject. He remains very active in education and training and has participated in numerous Conferences and Symposia. He also has received many honours and awards from many organisations for his work. 